\title{
Visual Impairment After Celecoxib Administration: A Rare Side Effect of a Commonly Prescribed Drug
}

\author{
Yasser Alwabli $^{*}$, Ahmed Alrasheedi ${ }^{2}$, Eid Alrasheedi ${ }^{2}$ and Fahad Alrasheedi ${ }^{2}$ \\ ${ }^{1}$ Assistant Professor of Orthopedic Surgery, Department of Orthopedic Surgery, Qassim University, College of Medicine, Qassim, Saudi Arabia \\ ${ }^{2}$ Medical Student, Qassim University, College of Medicine, Qassim, Saudi Arabia \\ *Corresponding Author: Yasser Alwabli, Assistant Professor of Orthopedic Surgery, Department of Orthopedic Surgery, Qassim \\ University, College of Medicine, Qassim, Saudi Arabia.
}

Received: September 03, 2019; Published: October 11, 2019

DOI: 10.31080/ASOR.2019.02.0110

\begin{abstract}
Introduction: NSAIDs are commonly prescribed family of drugs to control inflammation and pain. Celecoxib is a selective COX-2 inhibitor which was shown to be beneficial in reducing GI symptoms. In musculoskeletal conditions, Celecoxib has many indications, including osteoarthritis, rheumatoid arthritis, and other acute and chronic painful conditions. The side effects of celecoxib are almost similar to that of other NSAIDs. However, visual side effects are reported in the literature in a few cases. These range from inflammation of the conjunctiva to blurring of vision and temporary blindness. These effects are typically mild and self-limited. In this report, we present a case with rare visual side effects after celecoxib administration.

Clinical Case: A 42-year-old lady presented to the out-patient clinic with low back pain, right hip pain, and difficulty walking. The pain started after she gave birth two weeks before presentation. At first, she was investigated and started on Tylenol and then Celebrex to control her severe pain. She then developed ocular side effects in the form of temporary blindness and blurring of vision. She stopped Celebrex, and her vision improved. By the three-months follow up visit, she had complete recovery of her vision.

Discussion: The side effects of NSAIDs on the eyes are extremely uncommon. Clinicians should be aware of theses side effects and reassure patients about the prognosis of these symptoms. Although the side effects are temporary, an eye examination should be performed by an ophthalmologist to rule out concomitant pathologies.
\end{abstract}

Keywords: Celecoxib; Visual Side Effect; Blurring of Vision; Temporary Blindness

\section{Introduction}

Celecoxib is significantly more selective for cyclooxygenase-2 (COX-2) inhibition than that of cyclooxygenase-1 (COX-1) inhibitors. This selectivity against COX-2 provides a therapeutic advantage over the non-selectivity of COX-1 inhibitors. The proper management of chronic inflammatory conditions, by COX-2 inhibitors, is remarkably better [1]. Celecoxib has similar efficacy to NSAIDs in the treatment of pain, inflammation, and fever. This is thought to be caused by the inhibition of prostaglandin synthesis during inflammatory pathway [2]. Celecoxib, when used without concomi- tant aspirin therapy, is associated with less GI bleeding, dyspepsia, and fewer endoscopic ulcers than most other NSAIDs [4].

Celecoxib has many indications in managing many musculoskeletal conditions. It is approved for the treatment of osteoarthritis (OA), rheumatoid arthritis (RA), juvenile rheumatoid arthritis (JRA), and acute mild to moderate pain [1-4]. For osteoarthritis, Celecoxib has demonstrated a significant reduction in joint pain compared to placebo. Additionally, Celecoxib was evaluated for the treatment $\mathrm{OA}$ of the hip and knee. In one study, approximately 4,200 
patients were involved in placebo- and active-controlled clinical trial for up to 12 weeks duration. Administration of Celecoxib $100 \mathrm{mg}$ twice daily or $200 \mathrm{mg}$ once daily resulted in improvement in WOMAC (Western Ontario and McMaster Universities) scores, which is a composite of pain, stiffness, and functional measures in OA patients [5]. This improvement in pain and functional scores has led to the increasing popularity in Celecoxib prescriptions for the management of $\mathrm{OA}$.

In the management of RA, Celecoxib has demonstrated a significant reduction in joint pain and swelling compared to placebo. Tornetta (2006) noted that clinical trials involving more than 2,100 patients in placebo- and active-controlled clinical trials of up to 24 weeks in the treatment of RA. Celecoxib was shown to be superior to placebo in these studies. Using the ACR20 Responder Index, an index involving clinical, laboratory, and functional measures used in RA patients [5,7]. Moreover, Celecoxib is frequently prescribed for juvenile rheumatoid arthritis. It is used for relief of signs and symptoms of pauciarticular and polyarticular JRA in patients above the age of two and less than 17 years of age $[6,7]$.

\section{Literature review of similar cases}

Several case studies have demonstrated that patients who were treated with selective COX-2 inhibitors, specifically celecoxib or rofecoxib, have presented with acute temporary ocular adverse effects. The two main side effects are blurred vision and conjunctivitis [8]. These studies suggest that these effects are mainly due to the inhibiting release of prostaglandins, which affects retinal blood flow and leads to visual disturbance [8-10]. Non-selective COX inhibitors, like ibuprofen, have also reported side effects that included blurred vision [11,12]; however, their occurrence did not seem as prevalent as with COX-2 inhibitors [9]. Here are the results of the main case studies relating to the side effects of COX-2 inhibitors.

One of the first cases that linked celecoxib to ocular adverse effects is for a 79-year-old female patient who was treated with selective COX-2 inhibitors to control her back pain [10]. The patient was on Prinzide for hypertension. She was also on Hyoscyamine and celecoxib twice a day for back discomfort in addition to a daily multivitamin. Except for celecoxib, she had been taking these medications for several years. She then presented with a visual abnormality, specifically orange spots detected in a regular eye examination. These visual abnormalities appeared two months after starting celecoxib. As other cases have shown, the impairment has subsided with the discontinuation of celecoxib-while the other medications were continued [10].

Another case study by Coulter., et al. (2003) describes ocular adverse effects in patients who were treated for musculoskeletal disorders with either celecoxib or rofecoxib. Patients ranged in age between 53-81 years old. A total of 7 patients complained from visual field defect and blurred vision throughout the duration of the treatment. Patients taking celecoxib included four females and one male. They presented with visual field defect, reduced vision, blurred vision, and visual abnormality throughout the duration of the treatment. Patients treated with rofecoxib included two male patients. They presented with temporary blindness and blurred vision. The onset of the adverse effects was one week or less in 4 of the patients. For one patient, the onset presented after each dose. All the patients recovered immediately once the medication was discontinued. This demonstrated that the condition was completely reversible once the drug was withdrawn. Moreover, the study kept track of these patients for the following seven months and found that the symptoms did not recur during that period [9].

Lastly, a review article by Fraunfelder (2006) that related to ocular adverse effects associated with COX-2 inhibitor analyzed 8 cases that reported visual abnormality, which included either orange spots and jellybean-like area of vision loss for patients taking celecoxib and temporary blindness for patients on rofecoxib. In other cases, patients complained from blurred vision, four of these were taking celecoxib, and one only was taking rofecoxib. All these symptoms resolved within three days after the drug was discontinued [8].

\section{Case Presentation}

A 42-year old female patient presented to our out-patient clinic with a complaint of low back pain, right hip pain, and difficulty walking. She is not known to have pre-existing medical conditions, and she is not on any medications. The patient was referred to our clinic from the neurology clinic as she first visited them for the same complaint. This incident happened after she gave birth two weeks before presentation. On further questioning, the patient was in severe pain (pain score 8 out of 10), and she was dependent on her ambulation on family members and using a wheelchair.

On physical examination, the patient was sitting comfortably on a wheelchair. When asked to leave the chair, she experienced severe pain and only walked for one step. Range of motion of the 
right hip was restricted due to pain. Another physical examination points were unremarkable. Neurologic examination of both lower extremities was normal.

We investigated the patient with lab and imaging modalities. Xray of the right hip was obtained and showed mild arthritic changes in the right hip joint and mild diastasis in the symphysis pubis. Otherwise, no significant abnormalities are detected. Also, we sent her for MRI of the lumbar spine and the pelvis. MRI reported as there is an inflammation at the sacroiliac joint with no other significant issues. At the same time, infection work-up to rule out infectious causes was also performed; this included cell counts, ESR, and CRP. All results came back normal.

The patient was breastfeeding her newborn and wishing to continue that despite her severe pain. We discussed with her the treatment options and started on Tylenol tablets only for pain. There was no significant improvement. One week later, the patient came back with continuing severe pain, and she decided to stop breastfeeding and started on Celebrex for pain control.

Three weeks later, the patient's symptoms improved, but she had a new complaint. The patient presented with unilateral visual disturbance in the form of one incident of temporary center-offield blindness and moderate blurring of vision. Our ophthalmology colleague saw her for this complaint and reported his examination as no abnormality explains these visual disturbances. We advised her to stop Celebrex and switch to other NSAIDs.

On further follow-up, she experienced good improvement in her symptoms. Her vision improved gradually with no blurring of vision. One-month and three-month visits to the clinic revealed that the patient recovered her vision entirely with no residual complaints.

\section{Discussion and Conclusion}

NSAIDs are commonly prescribed drugs for a variety of indications, especially for musculoskeletal complaints. The main indication for their use is pain control, through the anti-inflammatory properties they possess. For its COX-2 selectivity, celecoxib has a unique indication in patients with gastric complaints. Clinicians should be aware of the common side effects of NSAIDs as well as the less common side effects, such as visual impairment. The side effects on the eyes are temporary. This fact should be discussed with the patients and reassure them about the consequences they may encounter. Additionally, although these effects are transient, clinicians should also consult with their colleagues in ophthalmology to perform a complete eye examination and exclude other eye disorders with similar presentation.

\section{Bibliography}

1. Whalen K., et al. "Pharmacology Lippincott". 6th ed. Philadelphia: Wolters Kluwer (2015).

2. Katzung, Bertram G., et al. "Basic and Clinical Pharmacology". 13th ed. New York: McGraw-Hill Medical (2012).

3. Ritter J., et al. "A Textbook of Clinical Pharmacology and Therapeutics'. 5th ed. Hoboken: Taylor and Francis (2012).

4. Ebadi M. "Desk Reference of Clinical Pharmacology". 2nd ed. Boca Raton: CRC Press (2008).

5. "Drug label information" DailyMed - CELEBREX- celecoxib capsule (2019).

6. Lauren Tornetta and Carolyn L Yancey. Celecoxib Clinical REVIEW BPCA, USA: (2006).

7. Rappaport Bob. Summary of Clinical Review of Studies Submitted in Response to the Pediatric Written Request for NDA 20-998, Supplement 021. Division of Anesthesia, Analgesia and Rheumatology Products (2006).

8. Fraunfelder F. "Ocular Adverse Effects Associated with Cyclooxygenase-2 Inhibitors". Archives of Ophthalmology 124 (2006): 277.

9. Coulter D. "Celecoxib, rofecoxib, and acute temporary visual impairment”. BMJ 327 (2003): 1214-1215.

10. Lund B and Neiman R. "Visual Disturbance Associated with Celecoxib". Pharmacotherapy 21 (2001): 114-115.

11. Tullio C. "Ibuprofen-induced visual disturbance". American Journal of Health - System Pharmacy 38 (1981): 1362-1364.

12. Nicastro NJ. "Over-the-counter Ibuprofen Can Cause Visual Disturbances". AJN, American Journal of Nursing 90 (1990): 64.

\section{Volume 2 Issue 11 November 2019 (c) All rights are reserved by Yasser Alwabli., et al.}

